\title{
Solving “Antenna Array Thinning Problem" Using Genetic Algorithm
}

\author{
Rajashree Jain ${ }^{1}$ and G. S. Mani ${ }^{2}$ \\ ${ }^{1}$ Symbiosis Institute of Computer Studies and Research, (A constituent of Symbiosis International University) Model Colony, \\ Pune Maharashtra State 411016, India \\ ${ }^{2}$ Departmetn of Information Technology, JS College of Engineering, Handewadi Road, Hadapsar, Pune 411028, India
}

Correspondence should be addressed to Rajashree Jain, rajashree.jain@sicsr.ac.in

Received 13 April 2012; Accepted 11 August 2012

Academic Editor: Yongqing Yang

Copyright (C) 2012 R. Jain and G. S. Mani. This is an open access article distributed under the Creative Commons Attribution License, which permits unrestricted use, distribution, and reproduction in any medium, provided the original work is properly cited.

Thinning involves reducing total number of active elements in an antenna array without causing major degradation in system performance. Dynamic thinning is the process of achieving this under real-time conditions. It is required to find a strategic subset of antenna elements for thinning so as to have its optimum performance. From a mathematical perspective this is a nonlinear, multidimensional problem with multiple objectives and many constraints. Solution for such problem cannot be obtained by classical analytical techniques. It will be required to employ some type of search algorithm which can lead to a practical solution in an optimal. The present paper discusses an approach of using genetic algorithm for array thinning. After discussing the basic concept involving antenna array, array thinning, dynamic thinning, and application methodology, simulation results of applying the technique to linear and planar arrays are presented.

\section{Introduction}

An antenna array refers to two or more antenna elements that are spatially arranged and electrically interconnected to produce a directional radiation pattern. The electrical characteristics, orientations, and polarizations of the elements forming part of the array as well as the geometrical arrangement of the array and their interconnections determine the overall performance of the array.

Antenna arrays containing large number of elements are frequently used in radar, communication, astronomy, and other systems. In order to reduce the element count, cost, weight, power consumption, and heat dissipation in these systems, thinning is sometimes performed by removing a percentage of array elements according to a suitable strategy. For a fixed antenna size, the thinning produces antenna arrays much cheaper than completely filled arrays, in terms of both hardware and control complexity. Moreover, although by thinning the main lobe width may remain approximately unaltered, there will generally be a reduction in antenna gain and also loss of control over the radiation pattern outside the main beam.

Thinning can be considered as a tool for reducing total number of active antenna elements in an antenna array when the main beam is narrow and the demand on the control of radiations outside the main beam is modest.

The main concern in the design of such thinned arrays is to find an optimal set of element spacing's to meet array specifications based on current distribution among the antenna elements. Since the array factor of the thinned array is a nonlinear function of element spacing and there are an infinite number of combinations of element locations, the problem of optimizing the array pattern with respect to the element locations becomes nonlinear and complex. Thus, it is not easy to design a thinned array optimally. Further, optimal thinning becomes more difficult for "off-normal" scanning or for arrays requiring special radiation needs.

In the past, many approaches have been proposed for thinning. A variety of methods have been suggested for pattern synthesis of the nonuniformly spaced arrays, which 
include Poisson's sum formula [1], iterative least square [2], perturbation [3], dynamic programming [4], minimax [5] technique, and so forth. In recent years, thinning has been considered as a combinatorial problem. However, for larger arrays, the number of all possible combinations becomes large and increases exponentially with increase of number of array elements. Hence checking every possible combination to find the optimum one is nearly impossible. One needs a faster and more reliable method to find the optimum solution. Since no deterministic method can be found for array thinning in such large arrays, probabilistic methods have been attempted. These focus on density of "on" elements in different parts of array and its effects on far-field pattern [6]. In recent years, nongradient-based optimization methods such as genetic algorithms, ant colony technique and particle swarm optimization [7-10] have been found useful in solving such problems. The purpose of this paper is to study use of genetic algorithm for optimal thinning in large arrays including those requiring scanning or meeting specific radiation pattern requirements. The study also includes ways of implementing the thinning process dynamically in a large array.

The details of thinning considered as an optimization problem are discussed in Sections 2 and 3 deals with certain basic features of genetic algorithm. The problem of array thinning to be optimized by using SGA is formulated in Section 4. Simulation results after applying SGA for different thinned linear and planar arrays are presented in Section 5. Challenges involved while using GA for larger array and under changing conditions are discussed in Section 6. This section also proposes zoning technique, bulk array computation methods, concept of acceptable solution, and dynamic thinning programmer along with some simulation results. Section 7 has some conclusions.

\section{Thinning as an Optimization Problem}

An array will basically be thinned to meet an objective. The objective may be to lower the side lobe level outside the main beam or to meet a specific radiation requirement. In either case it becomes an optimization problem of minimizing the side lobe level or a cost function defined based on the difference between desired radiation and actual radiation. Any optimization method used, especially when the array size increases may have to face the following challenges.

2.1. Increased Solution Space. As the total number of antenna elements increase the solution space increases at a much larger scale. Table 1 depicts the relationship between solution space and $N_{\text {total }}$ for a linear antenna array symmetric from center for a thinning factor of $\mathrm{TF}=0.25$ (25\% inactive elements). Therefore exhaustive search of all combinations is practical for only small arrays.

2.2. Landscape of Solution Space. When the solution space becomes large, the likelihood of landscape of the solution space becoming complex also increases. Though the type of exact landscape would depend to a large extent on the
TABLE 1: Solution space for a symmetric array.

\begin{tabular}{lccc}
\hline No. of array elements $N_{\text {total }}$ & TF & $N_{\text {inactive }}$ & Solution space \\
\hline 8 & 0.5 & 4 & 6 \\
8 & 0.25 & 2 & 4 \\
32 & 0.5 & 16 & $12 \times 10^{3}$ \\
32 & 0.25 & 8 & $18 \times 10^{2}$ \\
100 & 0.5 & 50 & $1.3 \times 10^{14}$ \\
100 & 0.25 & 25 & $4.6 \times 10^{12}$ \\
200 & 0.5 & 100 & $1 \times 10^{29}$ \\
200 & 0.25 & 50 & $2.4 \times 10^{23}$ \\
\hline
\end{tabular}

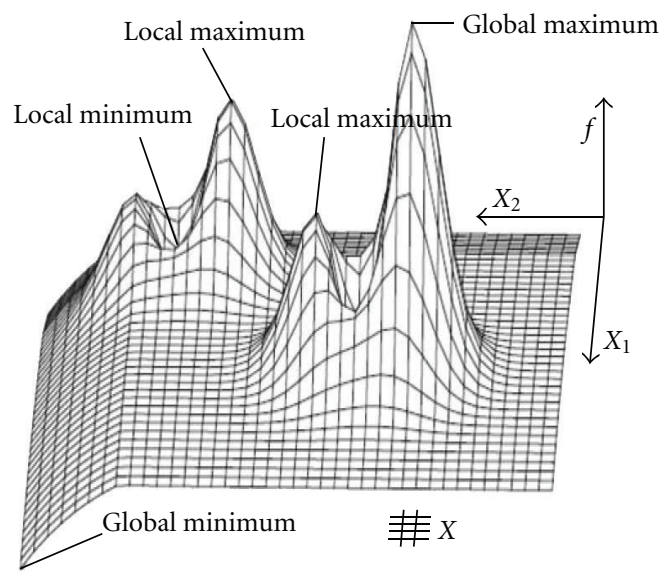

FIgURE 1: Typical landscape for optimization.

parameters of the array and the objective function to be maximized/minimized, its broad nature typically would be as shown in Figure 1.

2.3. NP Completeness. Although numerical techniques may provide a better thinned array design, the computer run time to solve the problem will increase exponentially as the array size increases. Thus array thinning can be categorized as discrete, combinatorial $N$-P complete optimization problem. Neither the analytical nor the gradient-based methods will provide a solution. Randomized search methods such as simulated annealing, genetic algorithm, particle swarm optimizer, ant colony optimizers have proven as more suitable. These algorithms are stochastic and are characterized by the following features:

(i) gradient free (do not use derivative information);

(ii) effective for multiobjective functions;

(iii) robust leading to practical acceptable solution rather than the best solution;

(iv) largely independent of initial design/solution domain;

(v) global techniques are useful when dealing with new problems in which area of the solution space is unknown. 


\section{GA for Thinning Antenna Arrays}

The concept of genetic algorithm was first formalised by Holland [11] and extended to functional optimisation by Goldberg [12], Davis [13], and De Jong [14]. It involves the use of optimisation search strategies patterned after the Darwinian notion of natural selection and evolution.

The major features of GA like direct use of coding, search from a population, blindness to auxiliary information and randomized operators contribute to its robustness and resulting advantage over other more commonly used techniques.

In particular, for solving electromagnetic problems and array thinning problems [15] GA has many advantages over the traditional numerical optimization approaches, including the facts that it

(i) can optimize with continuous or discrete parameters;

(ii) does not require derivative information;

(iii) simultaneously searches from a wide sampling of the cost surface;

(iv) can work with large number of variables;

(v) provides a list of optimum parameters, not just a single solution;

(vi) may encode the parameters and the optimization is done with the encoded parameters;

(vii) works with numerically generated data, experimental data, or analytical functions.

3.1. Anatomy of GA. There are many versions of genetic algorithms, one differing from another in some detail. In a nutshell, all genetic algorithms have two basic steps: during the selection step, a decision is made as to who in the population is allowed to produce offspring, and during the replacement step another decision is made as to which of the members from one generation are forced to perish in order to make room for an offspring to compete.

3.1.1. Simple GA (SGA). Simple GA (SGA) starts by randomly generating a population of $N$ individuals, that is, individual solutions. These individuals are evaluated for their fitness. Individuals with higher fitness scores are selected, with replacement, to create a mating pool of size $N$. This method of selection is called fitness proportionate reproduction (FPR). The genetic operators of crossover and mutation are applied at this stage in a probabilistic manner which results in some individuals from the mating pool to reproduce. The assumption here is that each pair of parents produces only one pair of offspring through the crossover operation. Now the population pool contains some individuals who never got a chance to reproduce and offspring of those who got a chance to reproduce. The procedure continues until a suitable termination condition is satisfied. All other versions of GAs are based on this simple form of GA or SGA.
3.1.2. Pseudo-Code and Flow Graph. Basic steps involved in implementing SGA are simple and straight forward. These are listed as follows and are discussed in subsequent paragraphs:
(a) genes, chromosomes, and coding a parameter set;
(b) create an initial population;
(c) evaluate fitness of each population member;
(d) invoke natural selection;
(e) select population members for mating;
(f) generate off-springs; Mutate selected members of the population;
(g) terminate or go to step b.
Simple flow graph for SGA is shown in Figure 2.

Genes, Chromosomes, and Coding a Parameter Set. The first step in using SGA is to code or map the parameters of the problem in hand into genes. Generally a parameter is equivalent to a gene. The coding is a mapping from the parameter space to the chromosome space that transforms the set of parameters to a finite length string of coded genes. Chromosomes can be entirely encoded in binary, floating point, or mixed binary and floating point.

Creating Initial Population. The initial population is the starting matrix of chromosomes. Generally each row of the matrix represents a set of variables forming part of a solution. An initial value of this set is normally taken as a random set within the limits as applicable for the variable. Depending on the coding of the parameter set, the elements of the starting matrix as stated before may be in binary, floating point, or mixed binary and floating point.

Fitness Evaluation. Each row of the starting matrix, representing a possible solution, is now evaluated for its suitability. For this, a cost function is to be chosen based on the problem in hand. Formulating the cost function is an important task, since this holds the key for the usefulness of the algorithm and its rate of convergence. This is chosen based on the goal(s) which need to be optimized. For multiobjective optimization, each cost can be normalized and weighed separately before combining them together to provide a single scalar quantity.

Natural Selection. The essence of the algorithm lies in promoting healthy members of the population to grow and yield off-springs, which are expected to be healthier. For this to happen, "survival of the fittest" theory is used. There are two common ways to invoke natural selection. The first method is to keep healthy chromosomes and discard the rest. For this, the population is sorted according to their fitness or cost and required elitist population is retained. A second approach based on thresholding can also be adopted, where all chromosomes having fitness above a predetermined value are retained. Thresholding method avoids sorting, which may be an involved process sometimes. The chromosomes 
that survive form the mating pool are then used for creating next generation of population.

Mate Selection. Mating is done among the most fitting members of the population based on probability. "Better the fitness, more chances of mating" is the basic principle followed. For this, two approaches are popular: Roulette wheel and Tournament selection.

For Roulette wheel selection, population is sorted and assigned a probability of selection on the basis of its rank in the sorted population. Chromosomes with low costs have a higher percent chance of being selected than do the chromosomes with higher costs. This needs to be worked out every time new population is being created.

In the Tournament selection approach, two small groups of chromosomes are selected randomly from the mating pool. The chromosome with the lowest cost in each group becomes a parent. Two such parents are then mated for producing off-springs. The number of tournaments held depends on the total number of parents required to make up the pool.

Generating Off-Springs. Off springs are generated based on two basic genetic operators: crossover and mutation.

Cross over refers to creating offspring's from portions of each parent. A typical method of creating a crossover between a set of parents is by using a mask. The point of crossover is generated in each case randomly. It is also possible to use multiple point crossovers, where different parts of off-springs are taken from the two parents.

Mutation is to induce random variations in the population. This is just done by flipping the binary digit at the position of mutating.

It has been observed that by having these two basic genetic operators, it is possible to make the healthy couple produce healthy off-springs, which can ultimately lead to minimization of the objective function.

Termination Criteria. The generation process is continued till a termination criterion is satisfied. This can be either based on number of total iterations or the achievable cost.

Though GA is a simple and robust algorithm, there are many things that can be implemented differently in various problems. First question is how to create chromosomes and what type of encoding to choose. Selecting GA parameters, population size, type and rate of crossover and mutation, selection criteria, and so forth, pays a critical role in achieving the results. All these depend on the final goal, the chosen cost function, and implementation of the algorithm.

GA optimisers, in general, are found to be robust, stochastic search methods. The powerful heuristic of GA is effective at solving complex, combinatorial, and related problems. They are particularly effective when the goal is to find approximate global maxima in a large-dimension, multimodal functional domain in a near-optimal manner. The next section of the paper discusses use of SGA for obtaining an optimally thinned array.

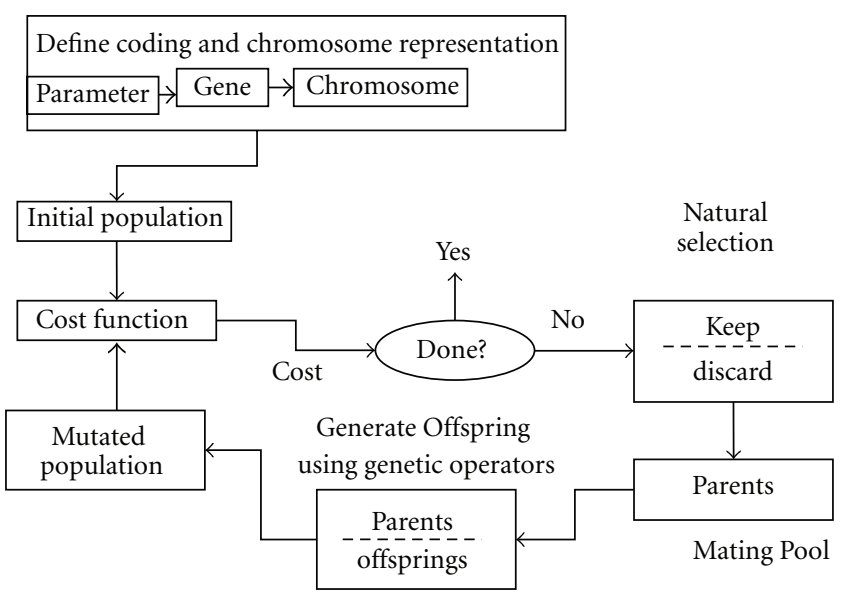

FIGURE 2: A block diagram of a simple genetic algorithm optimizer.

\section{Problem Formulation}

The array factor of a thinned array can be obtained by considering some of the elements as switched OFF from the uniformly excited array. Typical expression for far fieldintensity pattern from an antenna array of $M$ elements where the elements are simple and isotropic may be written as,

$$
F_{M}(\theta, \varphi)=\sum_{k=1}^{M} F_{k} e^{j \psi_{k}}
$$

where, $F_{M}(\theta, \varphi)$ refers to the radiation field in $(\theta, \varphi)$ direction, $F_{k}$ refers to the complex weightage of the antenna element $k, \psi_{k}$ refers to the complex weightage related to the array grid.

Where $F_{k}$ is either zero or unity according as the element is on (when the element is fed) or off (when the element is passively terminated in an impedance equal to the source impedance of the fed elements). Since the quantity $F_{k}$ can have only the values of 0 and 1 , we can say

$$
\begin{aligned}
F_{k} & =1 \text { if }\{\text { on }\} \in\{k\} \\
& =0 \text { else, }
\end{aligned}
$$

where $\{$ on $\}$ represent the subset of element numbers which are $\mathrm{ON}$.

By thinning, lesser number of antenna elements participate in the formation of the radiating beam in comparison to an unthinned array. Thinning factor (TF) is defined as

$$
\begin{gathered}
\mathrm{TF}=\frac{N_{\text {total }}-N_{\text {active }}}{N_{\text {total }}}, \\
N_{\text {inactive }}=N_{\text {total }}-N_{\text {active }} .
\end{gathered}
$$

Here $N_{\text {total }}, N_{\text {active }}$ and $N_{\text {inactive }}$ are total number of elements in the array and total number of active and inactive elements in the thinned array, respectively. Assuming a symmetrical linear antenna array, the problem thus transforms to finding the elements of the set $\{$ off $\}$ or $N_{\text {inactive }}$ in half array as shown if Figure 3 for a linear array and in the top right quadrant of a planar array as shown in Figure 4. 


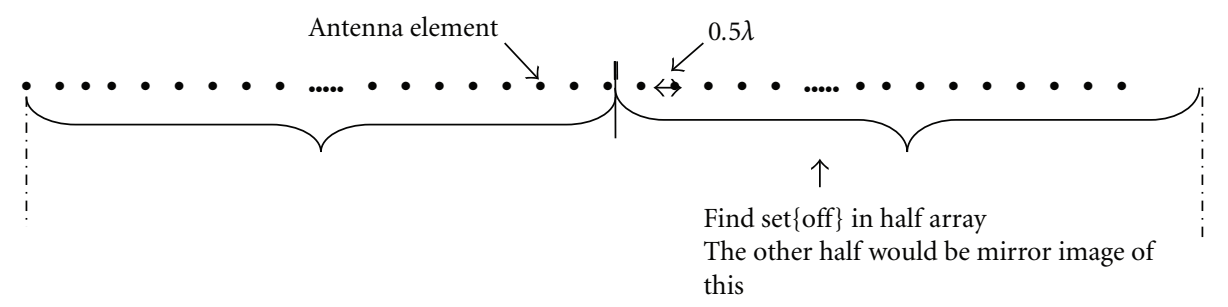

FIgURE 3: Symmetric linear array of $K$ antenna elements.

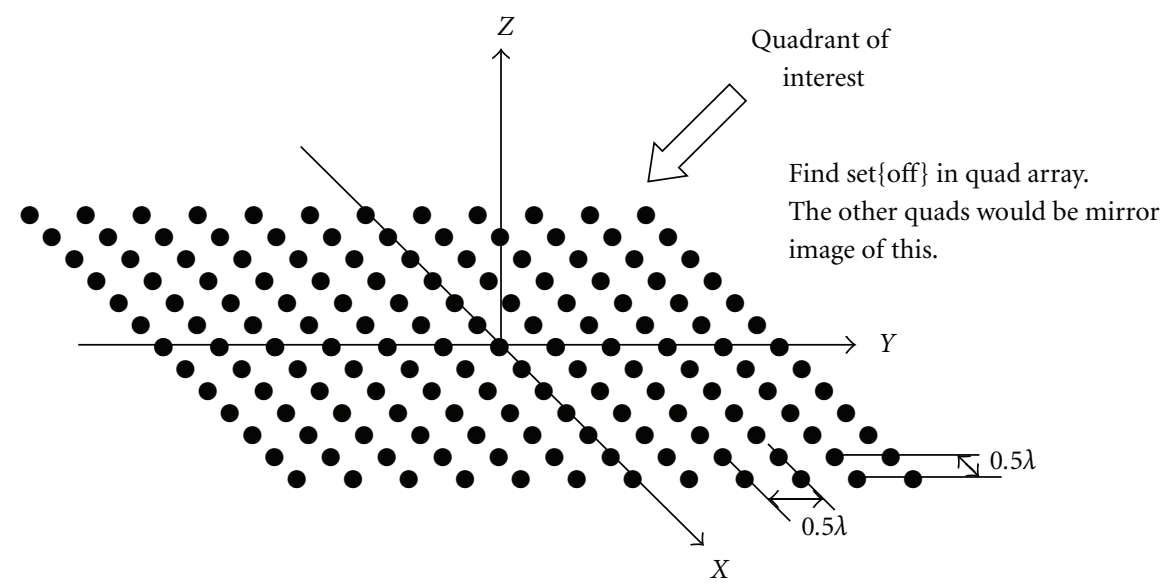

Figure 4: A planar array.

All studies related to the study of electromagnetic array antennas would be related to (1) expression in some form or other. It can easily be noticed that the above expression and all further relationships based on this expression tend to be complex and computationally intensive, especially when the number of elements are large and geometrically arranged in a different way. Also, since the function is not easily differentiable, optimisation using conventional analytical techniques becomes extremely difficult for large arrays.

\section{Application of SGA to Thinned Array}

As posed above, synthesis of a thinned array becomes a combinatorial problem of choosing a certain set ( $\left.N_{\text {inactive }}\right)$ out of an available set $N_{\text {total }}$ that the desired objective is satisfied. Approach using SGA for linear and planar arrays is discussed in the following paragraphs.

5.1. Coding and Initial Population. The first step of SGA optimization process involves coding of its parameters. For the thinned array problem, the best way of coding would require a " 1 " to represent an element which is "On" and a "0" to represent an element which is "Off". Any binary string of length $N_{\text {total }}$ will then represent a possible solution. A random set of such binary strings is taken as initial population. For example, for a 100 element linear symmetric array, a random initial population would mean 10 by 100 matrix of binary values, number of ones and zeros being decided on by the thinning factor (TF).
5.2. Fitness Evaluation. Fitness evaluation is based on the objective function (OF) requirement for thinning the antenna array. It can be of two types.

(a) It can be a cost function based on the variation between the envelopes of the desired radiation pattern and actual radiation pattern of the thinned array. In such a case, the purpose of design shall be to bring the variation below the set limit of $\varepsilon$ :

$$
\begin{aligned}
\varepsilon(\theta)= & \sum_{1}^{M}[\max \{(|F m(\theta)|-|F \max d(\theta)|), 0\}] \\
& +\Sigma[\max \{(|F \min d(\theta)|-|F m(\theta)|), 0\}],
\end{aligned}
$$

for an $M$ element array and where, $F \min d(\theta)$ and $F \max d(\theta)$ are the desired minimum and maximum radiation, respectively, and $\operatorname{Fm}(\theta)$ is the actual radiation in the direction of angle $\theta$.

(b) It can be any one of the following factors or a combination of them. It can also be a variation based on these factors:

$$
\begin{gathered}
\text { gain reduction factor, } \mathrm{GRF}=20 \log _{10}\left(\frac{N_{\text {total }}}{N_{\text {active }}}\right) ; \\
\text { side lobe variation factor, } \mathrm{SLVF}=(\mathrm{SL})_{M}-(\mathrm{SL})_{N} ; \\
\text { beamwidth variation factor, } \mathrm{BWVF}=(\mathrm{BW})_{M}-(\mathrm{BW})_{N} .
\end{gathered}
$$

Here $(\mathrm{SL})_{M},(\mathrm{SL})_{N}$ refer to the side lobe levels (in $\left.\mathrm{dB}\right)$ and $(\mathrm{BM})_{M},(\mathrm{BM})_{N}$ refer to the beam widths of the full and 


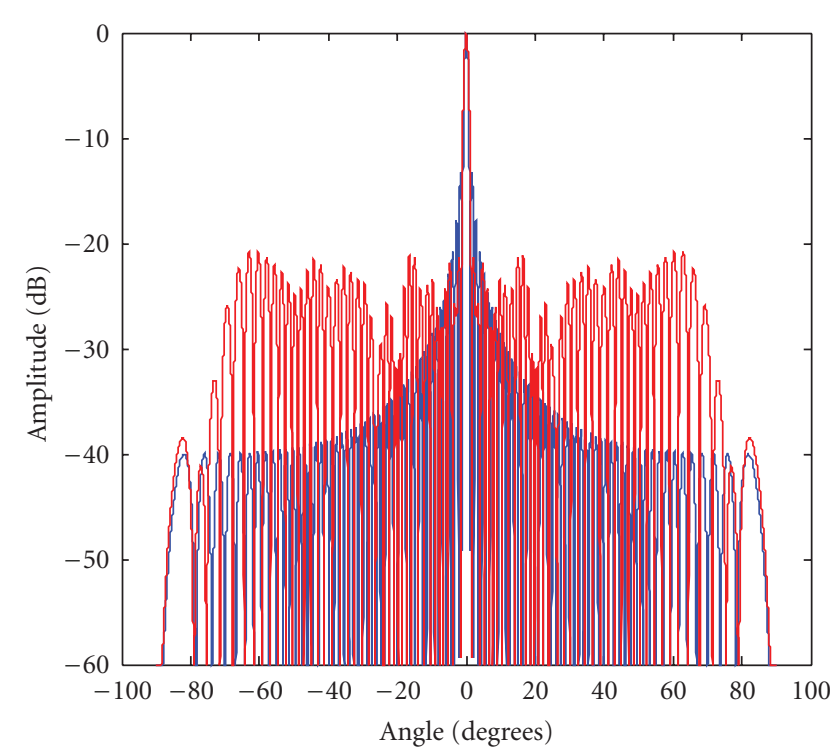

(a)

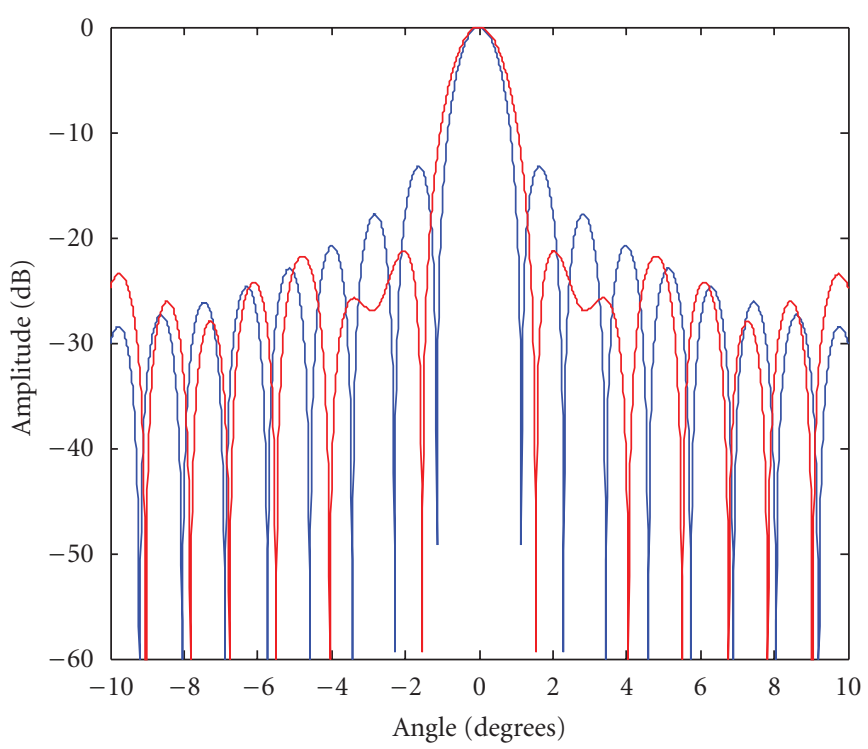

(b)

Figure 5: (a) Radiation pattern of a typical thinned array and (b) expanded view: Case 1 (blue: without thinning; red: after thinning).

thinned arrays, respectively. Depending on the type of OF, the procedure for thinning would aim to either maximize or minimize it.

Cost function is to be chosen based on the problem in hand. Since the major problem in a thinned array is its side lobe level, the natural choice for fitness evaluation shall be the side lobe level. However, this can be different based on the requirement.

The present paper makes use of cost function for some cases and the variation of peak side lobe level factor for the remaining cases.

5.3. Selection, Mating, and Termination. "Survival of the fittest" and "Better the fitness, more chances of mating" are the basic principles governing selection and mating process. For this, the side lobe level corresponding to each binary string in the population is computed, based on which the population is sorted.

Following the elitist rule, top half of the population is retained, discarding the rest. Mating is then carried out by following simple single point crossover technique. Mutation is the next step based on desired probability (0.02) of mutation. Thus the next generation of population is created where each member represents a possible thinned array. Two termination criteria are possible: (a) limiting the number of iterations or calls to the computationally intensive procedure of calculating sidelobe level and looking for the best possible solution; (b) looking for the desired side lobe level and terminate iterations accordingly.

5.4. Design Studies. The following four cases were considered.

Case 1. Peak side lobe level study.

Case 2. Limited angular region study.
Case 3. Limited angular region study.

Case 4. Optimizing the peak side lobe level in the two principal planes of $10 \times 20$ element planar array.

An array of 100 elements with a nominal interelement spacing of $0.5 \lambda$ was considered for Cases $1-3$. Since genetic algorithms are stochastic in nature, a number of runs were done in each case. Each run started with a new random set of population and followed the steps discussed above. For brevity, the discussion and results reported here are confined to only a limited number of runs.

5.4.1. Case 1: Peak Side Lobe Level Study. The objective of this case study was to examine suitability of SGA for reducing the Peak Side Lobe Level (PSLL) of a long linear array. The design exercise was carried out a number of times. In all cases, terminating criteria were restricted to the total number of side lobe calculations as 200 . In about $95 \%$ of the cases the peak side lobe had gone down from $-13.2 \mathrm{~dB}$ to better than $-20 \mathrm{~dB}$ within this restriction. About $20 \%$ reduction in number of elements could be achieved in most cases. The uniform array has a half power beam width of 1.01 degrees; thus the change in beam width due to thinning varies from $15 \%$ to $23 \%$. The uniform array has a beam width between nulls of 2.30 degrees; thus the change in null beam width due to thinning varies from $23 \%$ to $40 \%$. Figure 5 (a) shows one of the typical array radiation patterns of the final thinned array. Radiation pattern without thinning (uniform illumination) is also plotted for comparison. Figure 5(b) is an expanded version of Figure 5(a) near the main beam.

5.4.2. Case 2: Limited Angular Region Study. The objective of this case study is to examine if SGA can be applied for reducing the PSLL over a limited angular region. The angular 


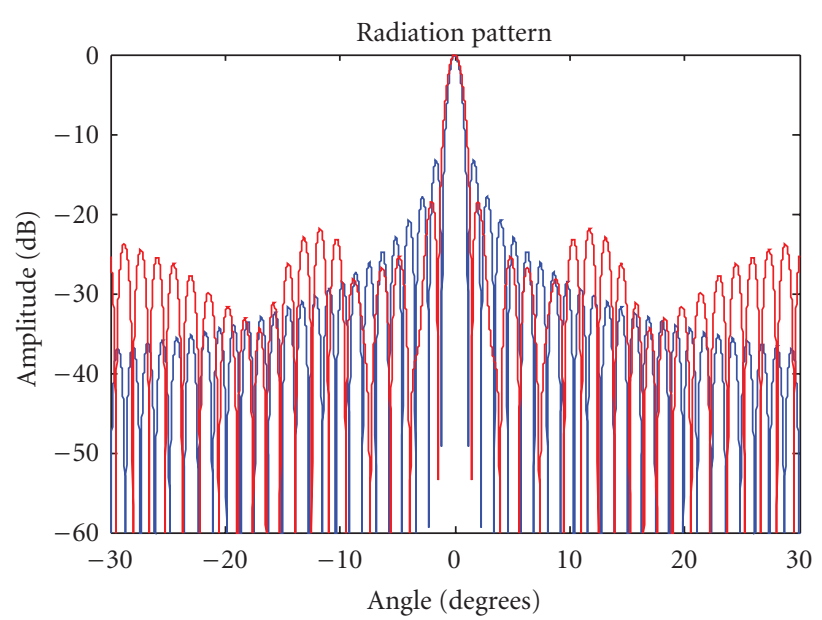

(a)

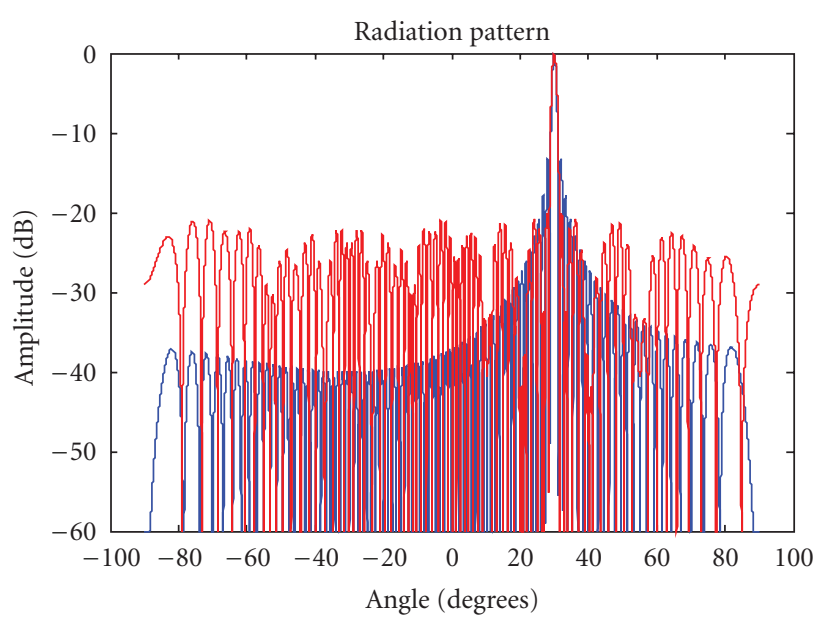

(b)

FIGURE 6: (a) Radiation pattern of a typical thinned array limited angular case, (b) radiation pattern obtained for 30 degrees scan angle. (blue: without thinning; red: after thinning).

region chosen was \pm 30 degrees on either side of beam maximum. It is evident from the results that the algorithm works effectively even for a narrower angular region. In every case, the side lobe level improves from $-13.2 \mathrm{db}$ to better than $-21 \mathrm{~dB}$ within the specified \pm 30 degrees. It is also interesting to note that the algorithm concentrates in the region of specific interest irrespective of the side lobe levels outside the region. In some cases the PSLL remains quite bad outside the region of interest. A typical radiation pattern are shown in Figure 6(a).

5.4.3. Case 3: Scanned Array Study. In this case, applicability of SGA for a linear scanned array is examined. The same array as considered in earlier cases was now scanned by providing a linear phase shift across its aperture, all other conditions being the same. Two scan angles of 30 and 45 degrees were considered. Typical results obtained for 30 degree scan angle are shown in Figure 6(b).

5.4.4. Case 4: $20 \times 10$ Element Planar Array. For this a planar array of 200 elements was chosen.

Configuration: rectangular: element grid: rectangular.

No. of elements along $x$-axis: $2 N x$ is 20 .

No. of elements along $y$-axis: $2 N y$ is 10 .

Total number of elements is 200 .

Normalized spacing along $x$-axis: $D x$ is 0.5 .

Normalized spacing along $y$-axis: $D y$ is 0.5 .

A solution of thinned locations of a quarter and another with full array is shown in Figure 7(a) and 7(b).

Figure 8 is a typical radiation pattern of 200 element planar array in its two principal planes. The objective here was also to reduce the peak side lobe level below $-20 \mathrm{~dB}$ value in both of its principal planes.

The four design studies discussed above gave us a confidence of planning to apply SGA to thin a large planar array of around 4096 elements under different scan conditions and also explore possible challenges for dynamic thinning. The challenges faced and the methodology the team has used to resolve these challenges are discussed in Section 6.

\section{Dynamic Thinning}

"Dynamic thinning" refers to response of the thinning process to a dynamic situation. This can be treated as on-line or real-time optimization process. Examples of two typical dynamic situations in respect of an antenna array case are as follow:

(1) to introduce a notch in a defined angular sector of the radiation pattern;

(2) the need to scan the main beam of the antenna array to a different angle

The optimization process should be able to respond quickly to the new demand. Sometimes the new demand may also arise after the optimization process has been initiated. This means that the requirements or constraints when the process of optimization started were different from the time solution for optimization was finally obtained. In such cases, sensitivity of the solution to the changed environment would decide the usefulness of the whole process.

6.1. Factors Affecting Dynamic Thinning. Dynamic array thinning refers to varying the thinning pattern on real time basis to suit to varying conditions. Even though SGA is a well-suited tool for solving array thinning problems, two factors figure prominently in applying the algorithm effectively for dynamic thinning.

(i) Computational complexity involved in thinned array design for many large size antenna arrays is too time consuming and hence may not be directly suitable for on-line implementation involving dynamic thinning.

(ii) In general, thinning procedure would aim to maximize/minimize the objective function (OF). But for a dynamic situation, it may often be sufficient if the OF can meet a specified level as governed by the operational requirements. This would mean that the design must aim at obtaining "an acceptable solution" rather than finding "the optimum solution". 


\begin{tabular}{|c|c|c|c|c|c|c|c|c|c|}
\hline 41 & 42 & 43 & 44 & 45 & 46 & 47 & 48 & 49 & 50 \\
\hline 31 & 32 & 33 & 34 & 35 & 36 & 37 & 38 & 39 & 40 \\
\hline 21 & 22 & 23 & 24 & 25 & 26 & 27 & 28 & 29 & 30 \\
\hline 11 & 12 & 13 & 14 & 15 & 16 & 17 & 18 & 19 & 20 \\
\hline 1 & 2 & 3 & 4 & 5 & 6 & 7 & 8 & 9 & 10 \\
\hline
\end{tabular}

(a)

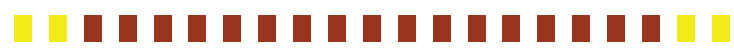

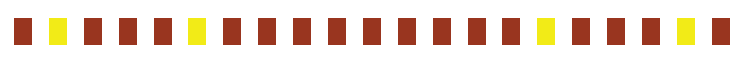

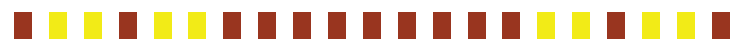

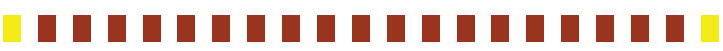

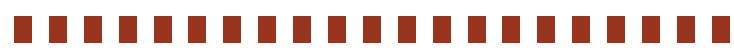

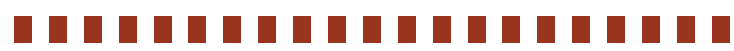

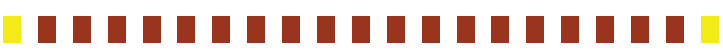
ロ

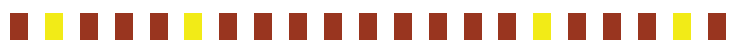

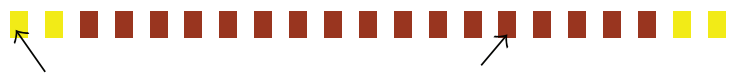
Inactive antenna element $(0)$

Active antenna element (1)

(b)

Figure 7: Examples of Typical Thinned solutions of $10 \times 20$ Element Array (a) Planar Array-Quarter-Highlighted element number is an inactive element position (b) Planar Array-Full.

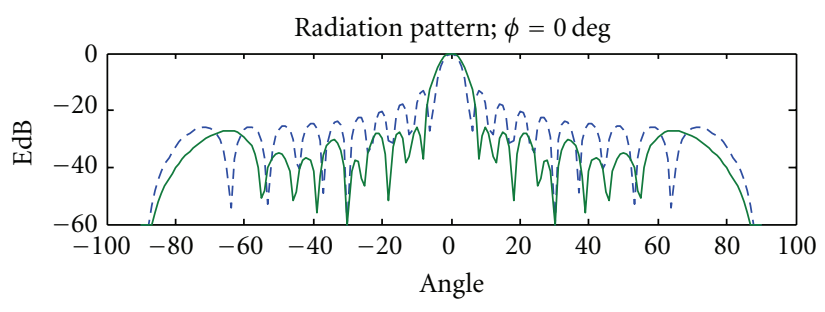

(a)

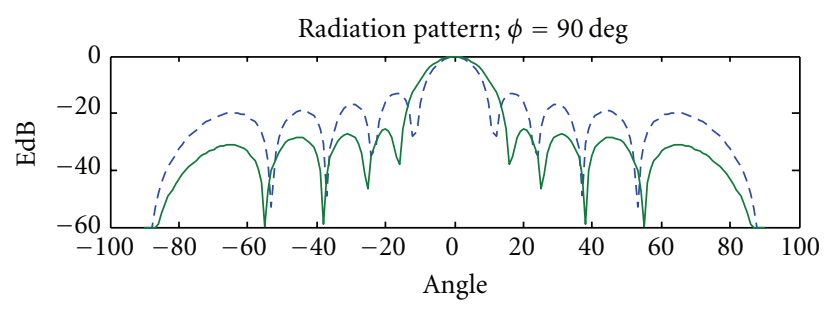

(b)

FIGURE 8: Planar array of $10 \times 20$ elements: radiation pattern: green Curve: thinned array and blue curve: uniform array.

6.2. Objective Function (OF) Requirement. This is as discussed in Section 5.2. Depending on the type of OF, the procedure for thinning would aim to minimize the cost function or obtain a predefined value of an objective function parameter.

6.3. Resolving Dynamic Thinning Issues. Since the major problem relates to computational complexity, efforts to reduce computational time and complexity were taken up as main issue. The following approaches were studied
(1) array symmetry;
(2) bulk array computation;
(3) zoning technique;
(4) thinning Simple Genetic Algorithm (TSGA);
(5) dynamic thinning programmer.

6.3.1. Array Symmetry. Fortunately several types of symmetry are common in practice in practical antenna arrays [16]. some of these symmetries can therefore be used to obtain a reduction in the computational complexity and solution space. In the present investigative study a linear array is considered as symmetric from center of the array, meaning the element excitations in the second half of the array would be mirror image of the excitations of the elements in the first half. Similarly for planar array quadrature symmetry was used.
6.3.2. Bulk Array Computation. Implementation of GA requires evaluation of $\mathrm{OF}$ for every member of the population in each iteration. Each of the evaluation is based on the array factor calculation $F_{M}(\theta)$ as mentioned in (1) for the linear array of $M$ identical elements. Similar equation in two dimensions needs to be evaluated for a planar array. These equations are highly nonlinear and involve lengthy procedures. Thus, for large arrays, requirements of computer resources for objective function evaluation would far exceed the functional requirements for SGA.

For dynamic thinning, special attention needs to be paid for the OF evaluation. The suggested method here is called as "Bulk Array computation" (BAC). This involves first storing the data of the radiated fields of all elements in all directions. The effect of "inactive elements" is then coalesced on the stored data of the radiated field of the array. Based on this, radiation pattern of the thinned array corresponding to each member of the population is then computed [17].

Major steps involved in the computation are

(1) generating data for creating "Element Table" which has all details about element location and its complex excitation coefficient;

(2) generating data for creating "Angle Table" which contains details of each angular direction in $(\theta, \varphi)$ coordinates; 
(3) computing and storing the radiated field due to each element of the array in each direction of interest;

(4) initial population of "inactive elements" is generated;

(5) effect of "inactive elements" is then coalesced on the stored data of the radiated field of the array; radiation pattern of the thinned array corresponding to each member of the population over the required angular sector is then computed;

(6) feedback parameter is extracted from the set of radiated patterns of the thinned arrays and is used in iterative manner to generate successive populations, using GA procedures;

(7) this is continued iteratively till the terminating criterion is obtained.

The flow graph for BAC is shown in Figure 9.

6.3.3. Zoning Technique. Zoning refers to partitioning the antenna array into convenient zones, so that the solution space can be usefully explored. Though there is no restriction in the total number of zones $N_{Z}$, each zone is expected to consist of at least 2 elements. $N_{Z}=1$ refers to no partitioning of the array. Figure 10 shows typical zoning of linear and planar arrays, where $N_{Z}=3$.

Generally, it is expected that number of inactive elements in the central portion (shown as zone 1 in Figure 10) of a thinned array would be much less than in other zones. Such a prior knowledge can help in partitioning of the array into zones and better exploitation of the solution space. In general, zoning is based on dividend or return likely to yield while exploiting the zone $[18,19]$. Though it is possible to partition into any number of zones, it may not be advisable to consider more than 2 or 3 zones, as shown later.

Zoning can help in enhancing convergence rate, since the proportion of the exploring space to total solution space reduces drastically in case of large arrays.

Adapting this technique for the present study involves generating initial population according to these criteria. Later SGA is carried out as in the previous study.

Zoning technique provides ample scope for using any a priori or intuitive information about the antenna array. By this approach considerable reduction in solution space occurs, resulting fast convergence as demonstrated in the next subsection.

6.3.4. Thinning Simple Genetic Algorithm (TSGA). For resolving the issues of computational complexity, increased solution space, zoning, symmetry consideration, cost function concept, and bulk array calculation methodology were integrated with SGA. The integrated method here is called as "Thinned Simple Genetic Algorithm" (TSGA). This involves first accepting the array details, domain details, and GA-related details. The standard SGA procedure involves objective function evaluation, sorting, natural selection, and reproduction using crossover and mutation, the population can be refined iteratively till it meets the required objective. A typical flow graph of TSGA is shown in Figure 11.

6.3.5. Dynamic Thinning Programmer. BAC and zoning techniques were found useful in reducing the overall computation time and help in achieving fast convergence required for dynamic thinning. SGA can be used for realtime thinning design by combining these techniques with a criterion based on an acceptable solution relevant to the dynamic requirement, as suggested earlier.

However, in case of larger arrays, the time response based on the above approach may not be adequate, due to operational constraints. In such cases, it is proposed that a dynamic thinning programmer (DTP) as shown in Figure 12 be used for system integration. DTP consists of a prestored data set, a dynamic thinning logic unit (DTLU), and a dynamic control circuit (DCC).

The prestored data set contains information about the elements of the set $\left\{N_{\text {active }}\right\}$ relevant for various conditions in the form of look-up tables. Based on operational requirements, appropriate trigger signals would be sent to the DTLU, which would retrieve information about the relevant $\left\{N_{\text {active }}\right\}$ set. For instance, in case of a scanning-phased array the trigger signal would be the scan direction information, which will enable DTLU to retrieve information about the on/off requirements of the array elements relevant for the required scanning conditions. These requirements would then be translated to appropriate control signals by the DCC and sent to the RF manifold for optimum thinning. By this process dynamic thinning can be achieved based on prestored data.

6.4. Simulation Results on Dynamic Thinning Issues. This section deals with some of the simulation results obtained using the methods suggested for dynamic thinning. In all cases, symmetrical arrays with isotropic radiators placed at a uniform spacing of half wavelength are considered. No mutual coupling effects are considered. The following case studies are discussed in the following sections:

(i) simulation results on zoning;

(ii) simulation results on BAC technique;

(iii) simulation results on scanning array.

6.4.1. Study on Effect of Number of Zones. To test the efficacy of zoning the following three studies were carried out

(i) to study effect of number of zones on speed of convergence;

(ii) to study effect of zoning for different objective functions;

(iii) to study different types of zoning.

Effects of Number of Zones. Effects of number of zones on a 200 element linear thinned array are presented here. Number 


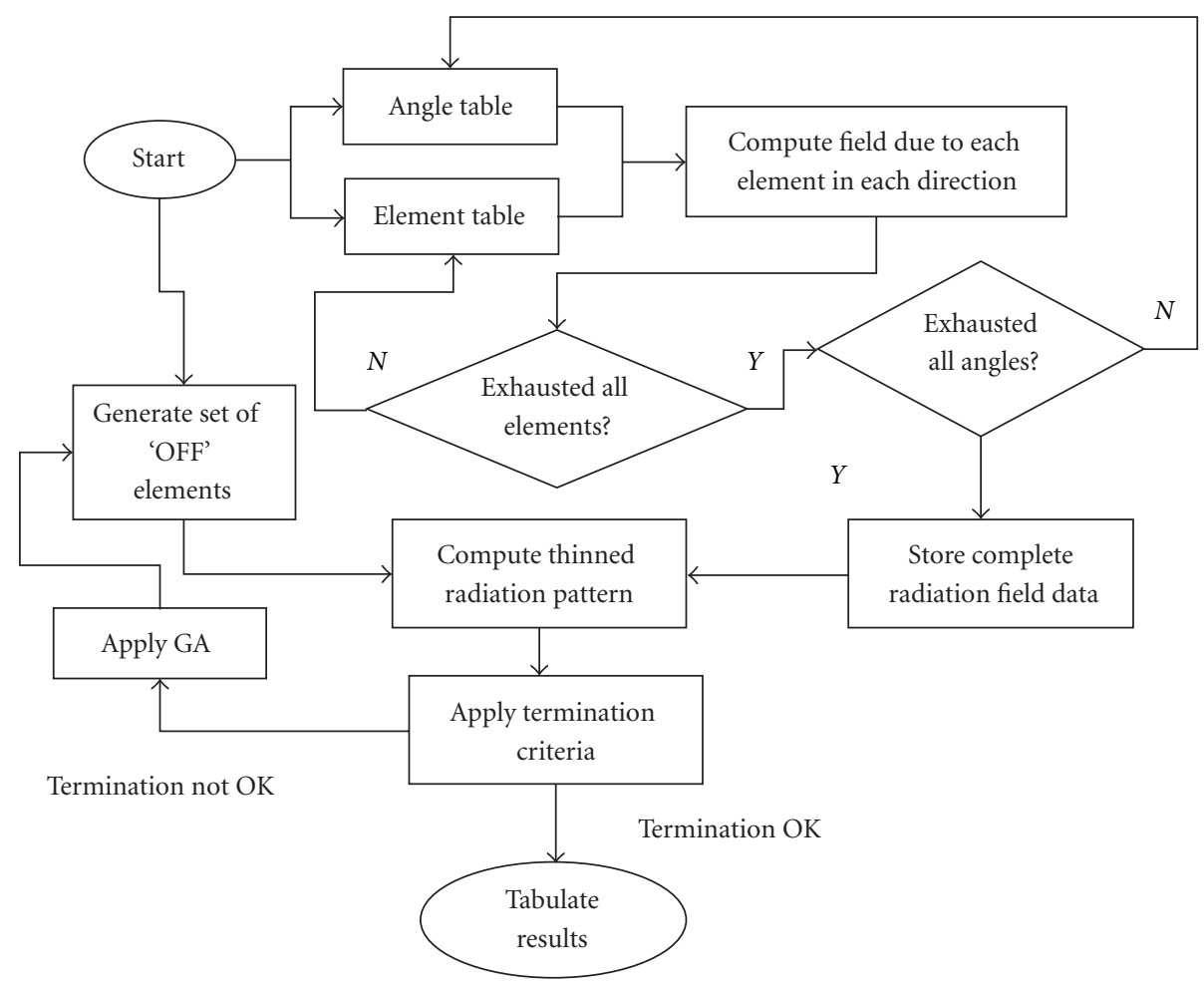

FIGURE 9: Flow chart for bulk array Computation.

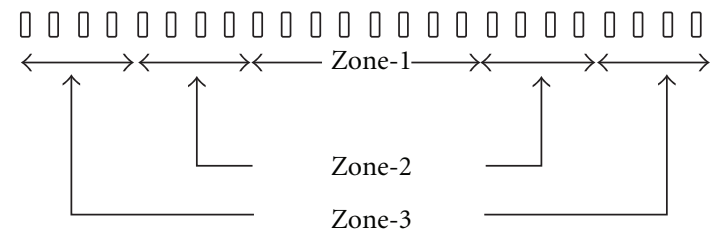

(a) Zoning of linear array

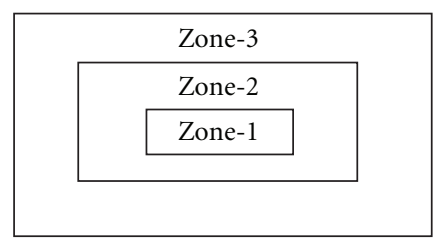

(b) Zoning of planar array

FIGURE 10: Zoning of antenna array.

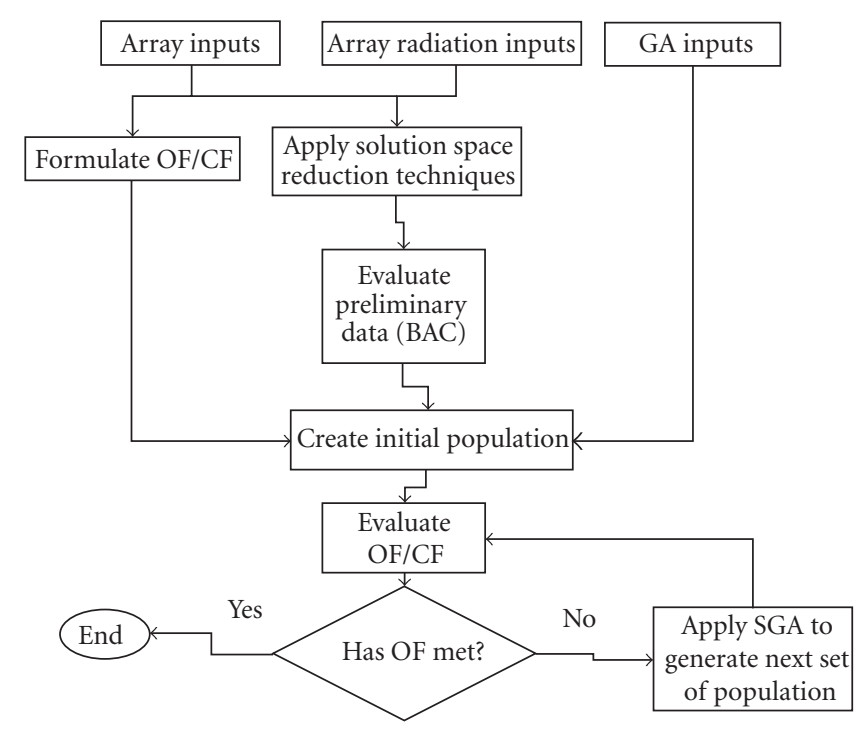

Figure 11: A flow graph for TSGA. 


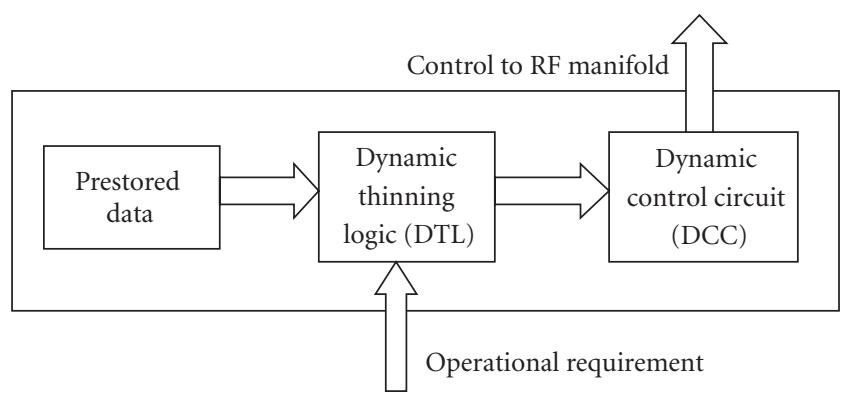

Figure 12: Dynamic thinning programmer.

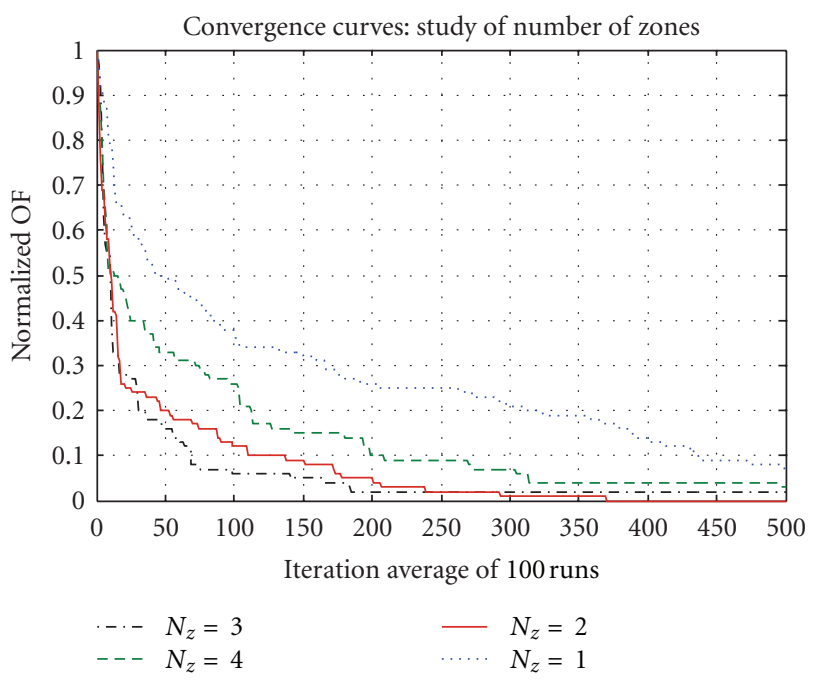

FIGURE 13: Convergence curves of study of number of zones.

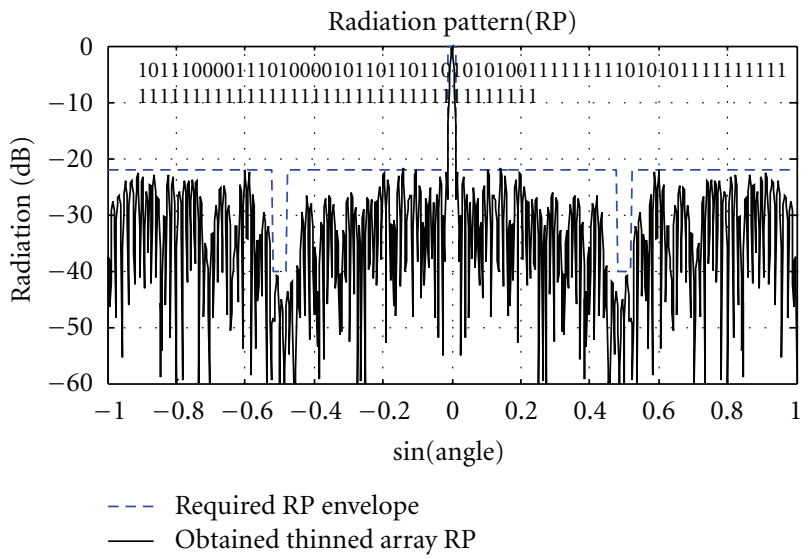

FIGURE 14: Typical radiation pattern of the thinned array for $N_{\text {total }}=$ 200 (Inset shows the active elements).

of zones considered are 1, 2, 3, and 4 numbered from center to the edge. The thinning factor, $\mathrm{TF}=0.24$ was considered, so that $N_{\text {inactive }}=48$. This is distributed in increasing proportion from Zone 1 to Zone $N$, so that Zone1 has least number of $N_{\text {inactive }}$ elements. It is ensured that symmetry is maintained in the thinned array. Objective function, OF, was chosen as achieving $-22 \mathrm{~dB}$ side lobe level outside the sector of \pm 0.01 radians. Maximum number of iterations is $N_{\text {it }}=500$. Terminating condition was to achieve OF or number of iterations $=N_{\mathrm{it}}$, whichever is earlier. In order to remove any possible biases due to stochastic nature of the algorithms and to obtain conclusive results, 100 runs were made in each case. Convergence behavior is shown is Figure 13.

It clearly shows zoning has helped converge the algorithm to an optimal solution especially the performance was better with 2 zones.

Study Effect of Zoning for Different Objective Functions. The OF was redefined to achieve radiation pattern envelope with a main beam sector and a notch sector. The results discussed are for linear arrays with $N_{\text {total }}=100$ and $N_{\text {total }}=200$. Main beam sector was taken as $\pm 0.02 / \pm 0.01$ radians for the two arrays. The notch sector was from \pm 0.48 to \pm 0.52 radians, with notch depth of $-36 \mathrm{db} /-40 \mathrm{~dB}$ for the two arrays. Symmetrical notch is due to array symmetry. Total number of iterations $\left(N_{\text {it }}\right)$ and total number of runs $\left(N_{\text {run }}\right)$ were taken as 500 and 100 , as before. Only $N_{Z}=1$ and $N_{Z}=$ 2 were considered. Cost function (CF) was defined based on the difference between the desired and actual radiation pattern based as per (4).

It was interesting to note that no significant improvement in performance could be noticed with zoning for both cases of $N_{\text {total }}=100$ and 200. Success rate was only 0 and $10 \%$ for the two cases. However, a cost reduction rate of 3.6 (from 20 to 5.5) was seen in case of average CF for $N_{\text {total }}=100$, which increases to 10.4 (from 60 to 5.74) for $N_{\text {total }}=200$. Thus, zoning can help in enhancing convergence rate, when the size of the array is larger. Moreover, it can also help in achieving an acceptable solution, rather than achieving an absolute optimized solution.

A typical radiation pattern when convergence had occurred for 200 element array is shown in Figure 14.

Study on Different Types of Zoning. For this study, a planar array with $20 \times 10$ elements was taken. The objective was to obtain $-20 \mathrm{~dB}$ side lobe levels in both planes. Three types of zoning were considered as shown in Figure 15(a). Convergence graphs with zoning are given in Figure 15(b). For this particular case, it can be observed that strategy 3 provides better convergence. 

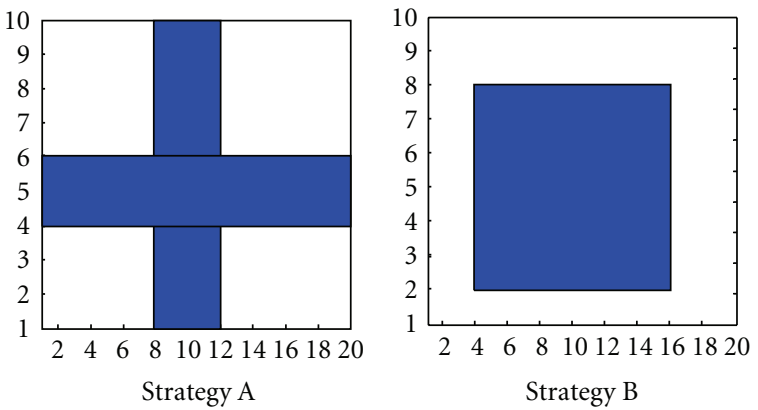

(a)

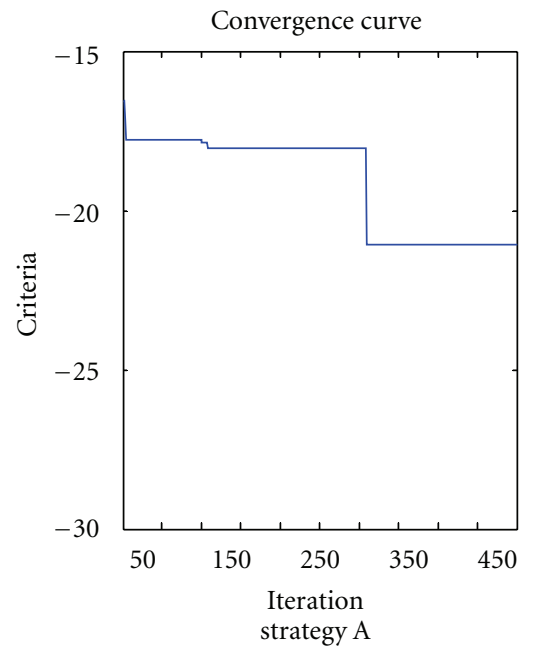

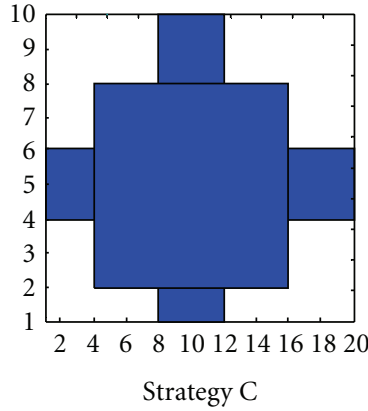

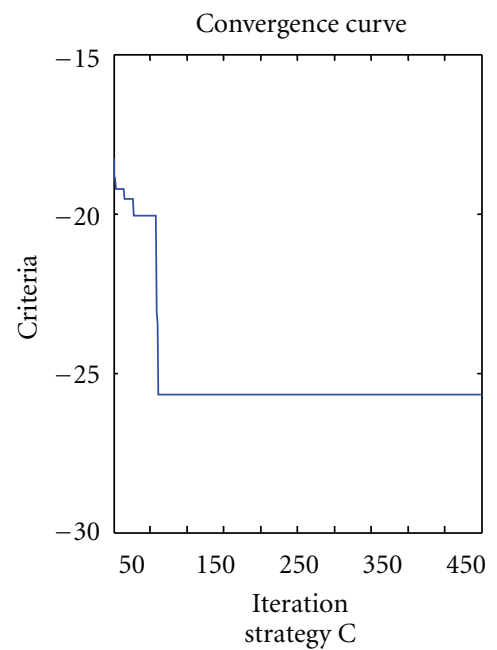

(b)

Figure 15: (a) Three types of zones on a planar array (blue: zone 1; white: zone 2), (b) respective convergence curves.

Convergence occurs, in all the three cases, thus showing that different types of zoning can be employed depending on the need. As discussed earlier, a priori knowledge can help in choosing the type of zoning most suitable based on the need.

6.4.2. Simulation Results on BAC Technique. As discussed earlier, bulk array computation' (BAC) is based on creating a table to store the data of the radiated fields of all elements in all directions and then subtracting the contributions from the "inactive" elements to obtain $\operatorname{FM}(\theta)$. There is an advantage by a factor of ( $1-\mathrm{TF} / \mathrm{TF})$ per each calculation of the array factor by following the suggested method.

For example for a symmetric planar array of $N_{\text {total }}=4096$ elements with a thinning factor of 0.38 , the present method would need $2 \times 10^{12}$ operations to evaluate the objective function as compared to regular array calculation method which would need $4 \times 10^{12}$. The angular directions considered for the example were $1025 \times 1025$ for a population size of 20 and the algorithm was run for 300 iterations. Clearly use of $\mathrm{BAC}$ technique is more suitable for large array thinning also under dynamic conditions.

6.4.3. Simulation Results on Scan Angle Study. Techniques discussed above were used to simulate thinning of a $64 \times$ 64 element planar array, scanned to different angles in the two planes. Techniques discussed above were able to select the right combination of 2784 elements within about 300 iterations in most of the cases. Typical results are shown in Figures 16(a)-16(f). Radiation pattern of the full array (without thinning) has been superposed for comparison. It was observed that the scanning behavior of the thinned array is on the expected lines. The beam maximums for the thinned and unthinned arrays coincide and occur at the expected angle in each case. The radiation patterns are smooth and do not have any grating lobes in spite of the thinning by about $32 \%$. Total no. of elements in one quadrant is 1024; no. of elements switched off in one quadrant is 328 .

\section{Conclusions}

The present paper discusses use of genetic algorithm for optimizing thinned linear and planar antenna arrays. It also discussed the challenges faced while applying simple GA to a larger array under dynamic conditions. Zoning and bulk array calculation methods helped GA to converge faster to an optimal solution. SGA was successfully applied to optimize scan performance of a large planar array of 4096 elements. Several design case studies are presented to prove the concepts. 

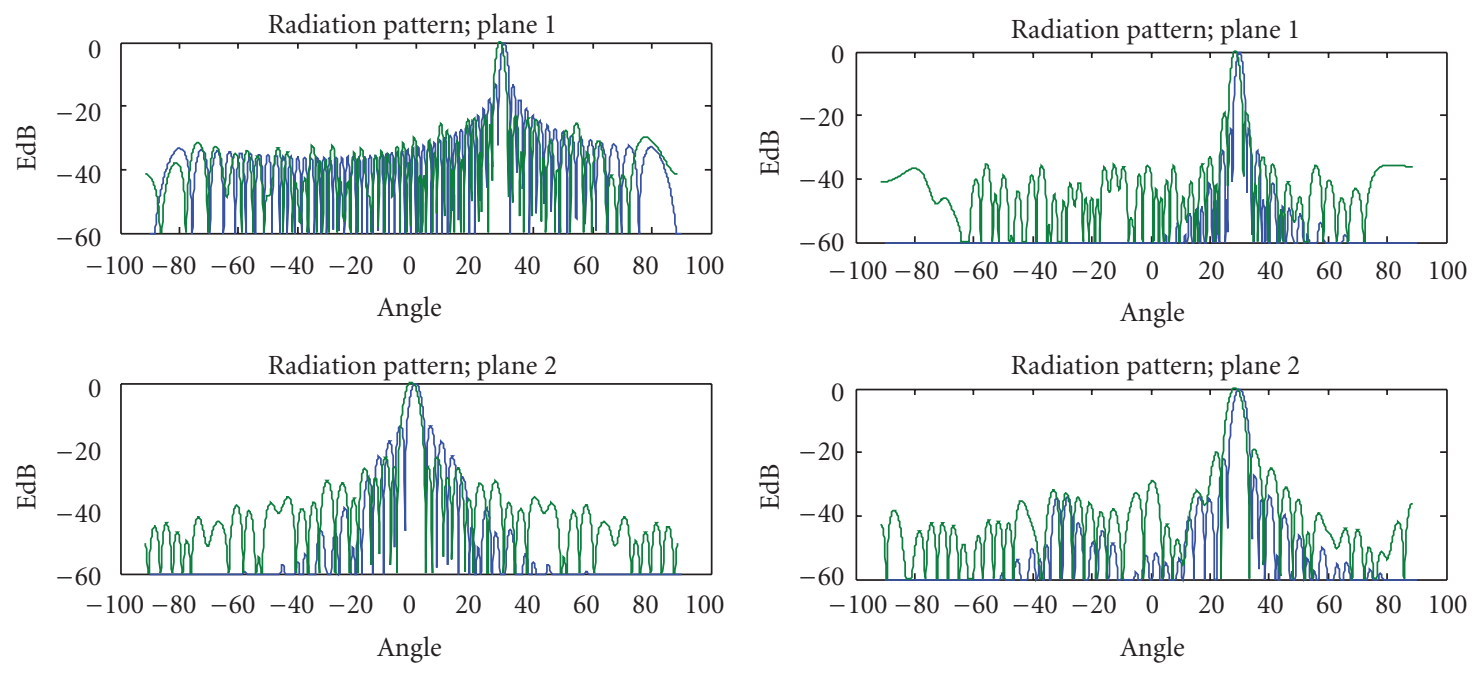

(a) Theta scan $=30$; Phi scan $=0$

(b) Theta scan $=30$; Phi scan $=30$
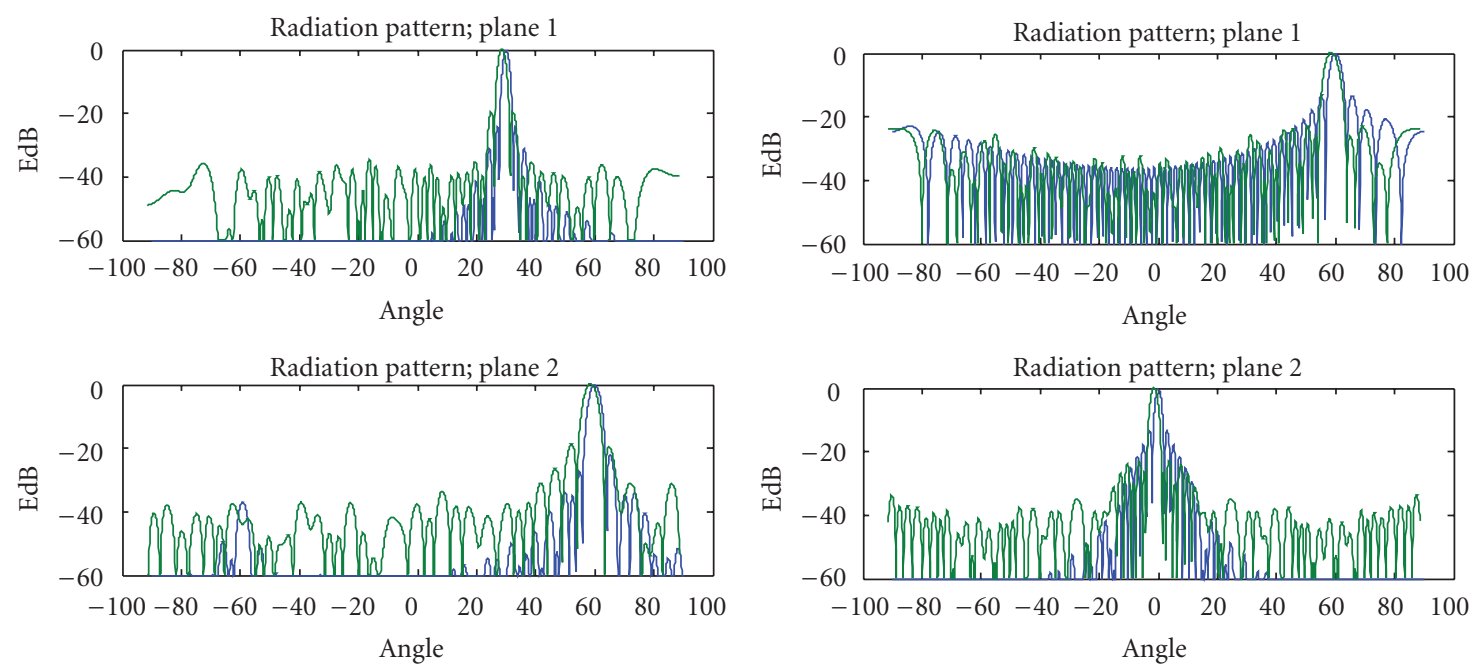

(c) Theta scan $=30$; Phi scan $=60$

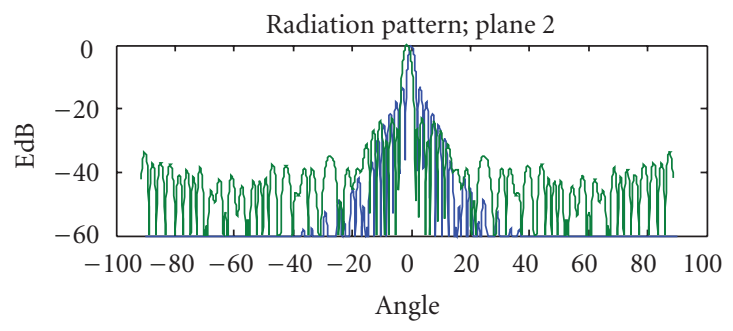

(d) Theta scan $=60$; Phi scan $=0$
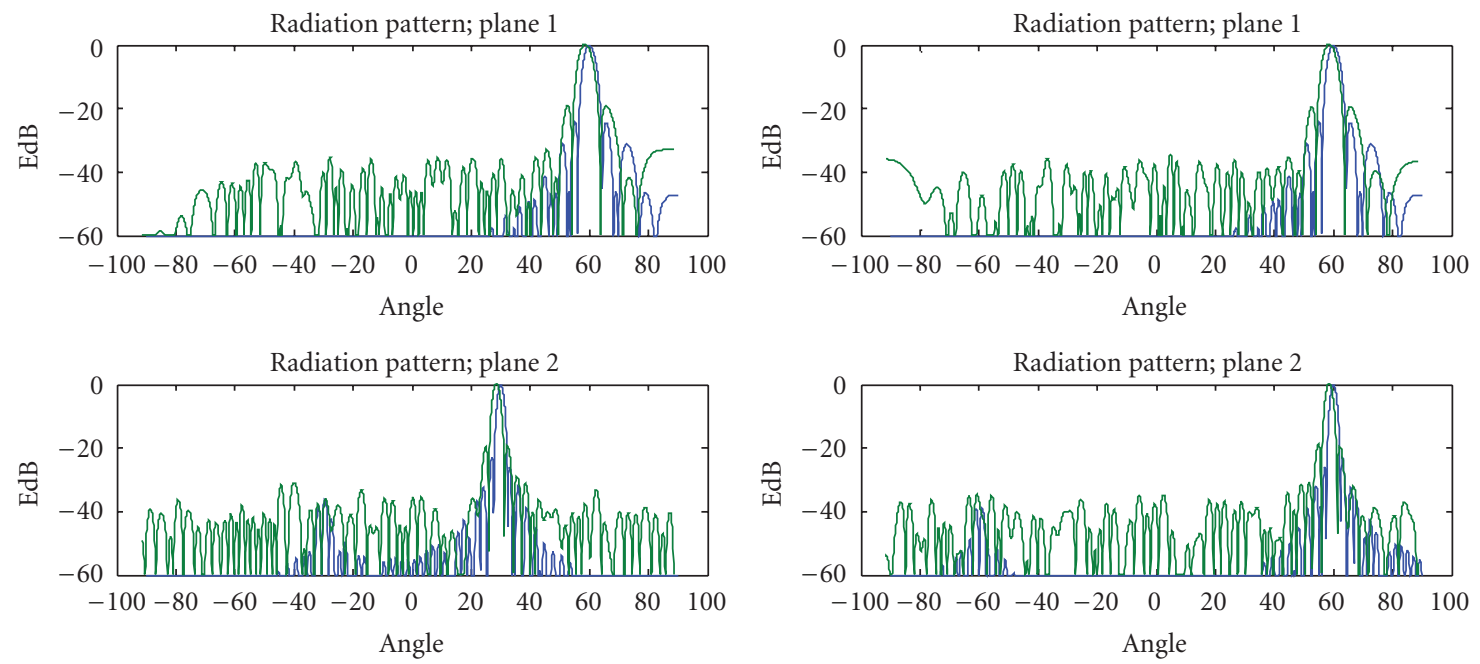

(e) Theta scan $=60$; Phi scan $=30$

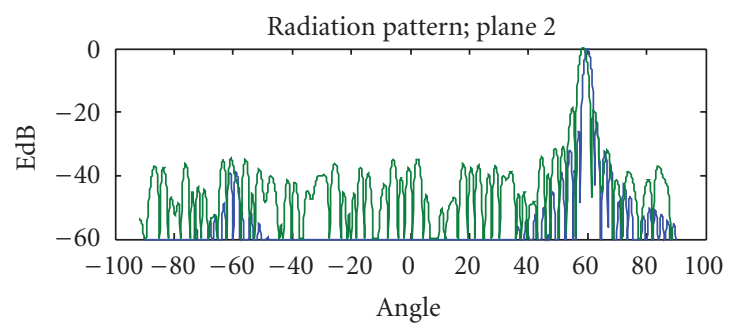

(f) Theta scan $=60$; Phi scan $=60$

FIGURE 16: Radiation patterns for different scanning conditions (inner full array; outer thinned array). 


\section{References}

[1] A. Ishimaru, "Theory of unequally-spaced arrays," IRE Transactions on Antennas and Propagation, vol. AP-10, pp. 691-702, 1962.

[2] X. Ma and B. K. Chang, "Weighted least square method for optimum thinned antenna arrays," in Proceedings of the IEEE Antennas and Propagation Society International Symposium, pp. 2232-2235, July 1997.

[3] M. G. Andreasen, "Linear arrays with variable inter-element spacings," IRE Transactions on Antenna and Propagation, vol. AP-10, pp. 137-143, 1962.

[4] M. I. Skolnik, G. Nemhauser, and J. W. Sherman, "Dynamic programming applied to unequally spaced arrays," IRE Transactions on Antenna and Propagation, vol. AP-10, pp. 35-43, 1964.

[5] B. K. Chang, X. Ma, and H. B. Sequeira, "Minimax-maxmini algorithm: a new approach to optimization of the thinned antenna arrays," in Proceedings of the IEEE Antennas and Propagation International Symposium, pp. 514-517, June 1994.

[6] M. Skolnik, J. Sherman III, and F. Ogg, "Statistically designed density-tapered array," IEEE Transactions on Antennas and Propagation, vol. 12, no. 4, pp. 408-417, 1964.

[7] R. L. Haupt, “Thinned arrays using genetic algorithms," IEEE Transactions on Antennas and Propagation, vol. 42, no. 7, pp. 993-999, 1994.

[8] R. L. Haupt and D. H. Werner, Genetic Algorithms in Electromagnetics, John Wiley \& Sons, New York, NY, USA, 2007.

[9] Ó. Quevedo-Teruel and E. Rajo-Iglesias, "Ant colony optimization in thinned array synthesis with minimum sidelobe level," IEEE Antennas and Wireless Propagation Letters, vol. 5, no. 1, pp. 349-352, 2006.

[10] M. M. Khodier and C. G. Christodoulou, "Linear array geometry synthesis with minimum sidelobe level and null control using particle swarm optimization," IEEE Transactions on Antennas and Propagation, vol. 53, no. 8, pp. 2674-2679, 2005.

[11] J. H. Holland, Adaptation in Natural and Artificial Systems, University of Michigan Press, Ann Arbor, Mich, USA, 1975.

[12] D. E. Goldberg, Genetic Algorithms in Search, Optimization, and Machine Learning, Addison Wesley Longman, New York, NY, USA, 1989.

[13] L. Davis, Handbook of Genetic Algorithms, Van Nostrand Reinhold, 1991.

[14] K. A. De Jong, An analysis of the behaviour of a class of genetic adaptive systems [Ph.D. thesis], University of Michigan, Ann Arbor, Mich, USA, 1975.

[15] Y. Ramat-Samii and E. Michielssen, Electromagnetic Optimization by Genetic Algorithms, John Wiley \& Sons, New York, NY, USA, 1999.

[16] E. N. Gilbert and S. P. Morgan, "Optimum design of directive antenna array," The Bell System, Technical Journal, vol. 34, pp. 637-663, 1955.

[17] M. Fernández-Delgado, J. A. Rodríguez-González, R. Iglesias, S. Barro, and F. J. Ares-Pena, "Fast array thinning using global optimization methods," Journal of Electromagnetic Waves and Applications, vol. 24, no. 16, pp. 2259-2271, 2010.

[18] R. Jain and G. S. Mani, "Applying micro GA concept for problems with large and rugged solution space," in Proceedings of the IEEE Region 10 Conference (TENCON '09), November 2009.
[19] R. Jain and G. S. Mani, "Dynamic Thinning of antenna array using genetic algorithm," PIERB: Progress in Electromagnetic Research B, vol. 32, pp. 1-20, 2011. 

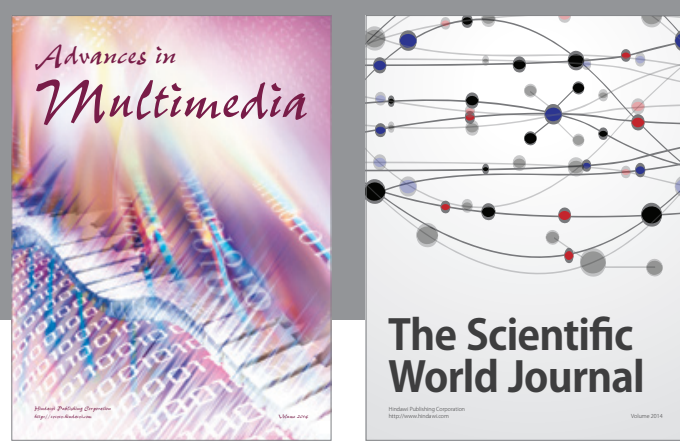

The Scientific World Journal
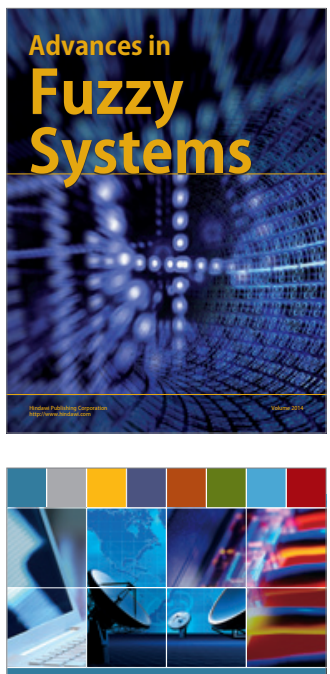

Computer Networks and Communications
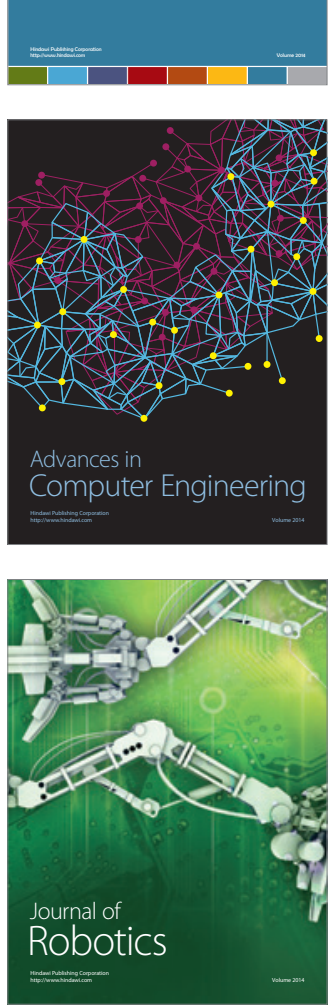
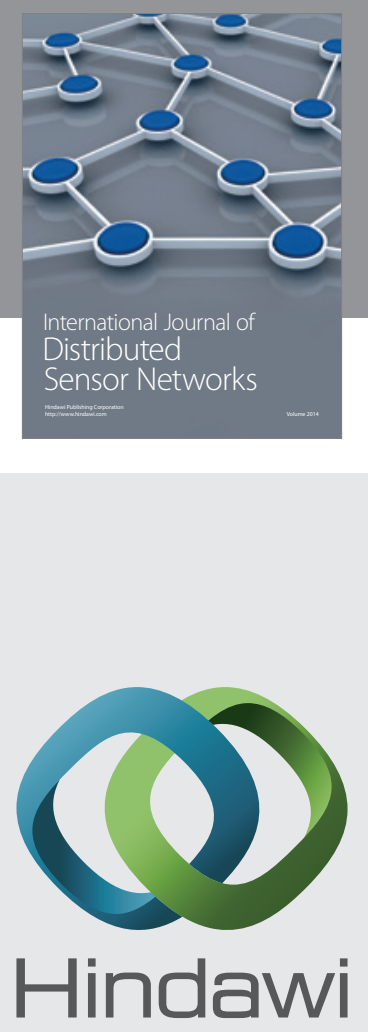

Submit your manuscripts at

http://www.hindawi.com
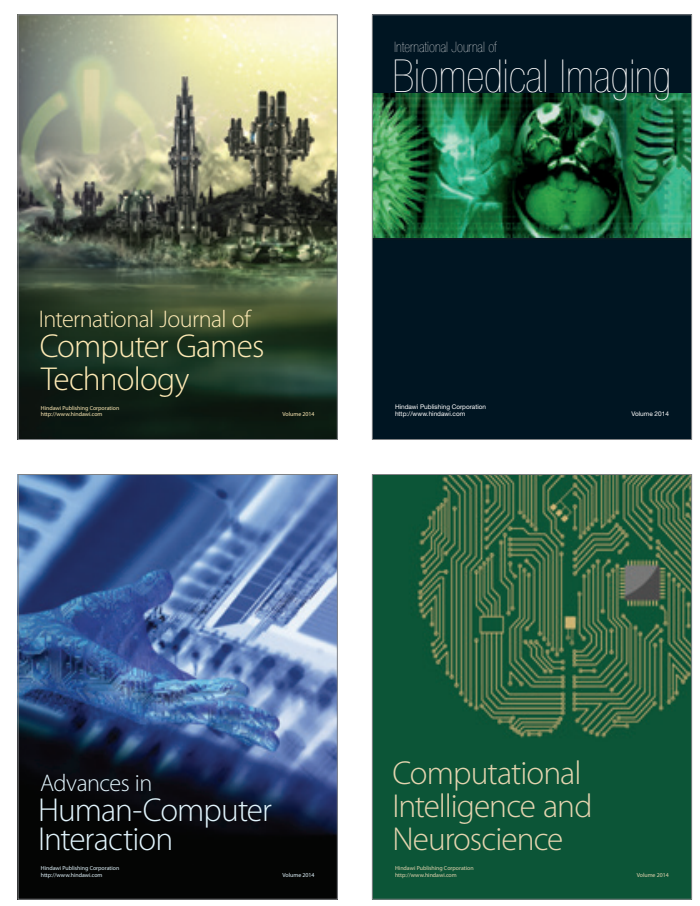
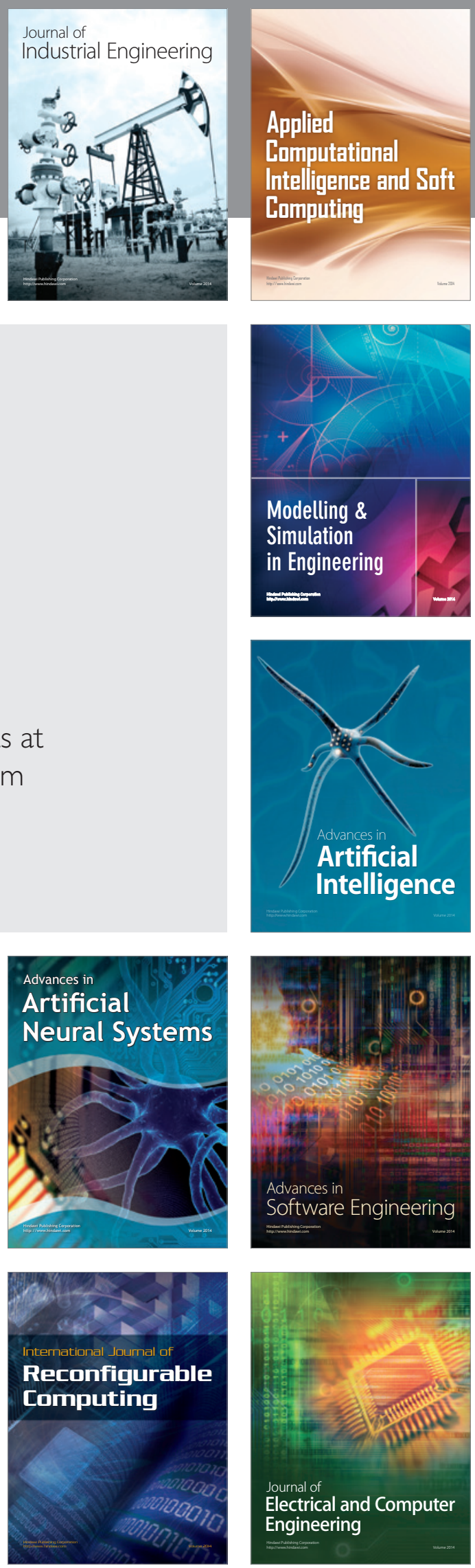